\title{
Photopolymerization Kinetics of Methacrylate Dental of Resin by Transmission FTIR Method and Reliable Methodology
}

\author{
Mirosław Kwaśny and Aneta Bombalska* \\ Institute of Optoelectronics, Military University of Technology, S Kaliskiego 2 str, 00-908 Warsaw, Poland
}

*Corresponding author: AnetaBombalska, Institute of Optoelectronics, Military University of Technology, S Kaliskiego 2

str, 00-908 Warsaw, Poland

\section{ARTICLE INFO}

Received: 幽 August 28, 2020

Published: 蔧 September 07, 2020

Citation: Mirosław Kwaśny, Aneta Bombalska. Photopolymerization Kinetics of Methacrylate Dental of Resin by Transmission FTIR Method and Reliable Methodology. Biomed J Sci \& Tech Res 30(1)-2020. BJSTR. MS.ID.004896.

\section{ABSTRACT}

The article presents the most reliable methodology for measuring the degree of conversion (DC) dental of resins in real time. It involves studying the kinetics of polymerization of the optical resin layer sandwiched between $\mathrm{KBr}$ crystals. Spectra of resins monomers and polymers were recorded from the parallel optical layers (about $0.15 \mathrm{~mm}$ thickness) of samples placed between the $\mathrm{KBr}$ crystals.

\section{Introduction}

The degree of conversion of monomer in dental resin-based composites (RBCs) is a very important parameter and is still the subject of a large amount of contemporary work, e.g. [1-3]. The physical and mechanical properties of photo-cured RBCs are directly influenced by DC. The cured compositions based on the derivatives of methacrylate's exhibit a considerable number of remaining double bonds. In general, RBCs reach a DC ranging from $50-70 \%[1,3]$ to even $94 \%$ depending on irradiance, exposure time (or dose), composite composition, temperature, type of activator or light source. Such a wide spread of DC values of even similar, typical materials is caused not only by different parameters of the polymerization process but also by different DC measurement methods and not very repeatable procedures.The current DC determination methods are based on a comparison of the absorption bandwidth typical for carbon double bonds $(\mathrm{C}=\mathrm{C})$ monomer and one of the carbon single bond reference bands. The most frequently used reference absorption band has a wavenumber of $1608 \mathrm{~cm}^{-1}$ although another peak maximum e.g. 1730 or $4623 \mathrm{~cm}^{-1}$ are also used [4]. The method is correct if this reference band shows the same intensity over the entire DC range. This, however, as we have shown, is not accurate for any methyl methacrylate derivative. Even for pure methyl methacrylate, the $1638 \mathrm{~cm}-1$ and $1608 \mathrm{~cm}-1$ bands are not sufficiently separated.

\section{Materials and Methods}

The materials used in this study is Enamel Plus HRi (UE2). The collected sample was placed centrally on the $\mathrm{KBr}$ crystal (diameter $25 \mathrm{~mm}$, thickness $3 \mathrm{~mm}$ ). On the edge of the crystal a spacer and a second $\mathrm{KBr}$ crystal were placed. Then the crystals were slowly press to the set thickness of $0.15-0.2 \mathrm{~mm}$ (equal thickness ofspacer) in a typical matrix for preparing pellets and then placed in the holder. DC was measured in real time by a Fourier transform infrared spectroscopy (Spectrophotometer IS50 Thermo Scientific). in transmission mode after photoinitiation The FTIR spectra were recorded in real time for $25 \mathrm{~min}$, with two spectra per second. During the entire study, the same thin layer of material was placed between $\mathrm{KBr}$ crystals. 


\section{Results and Discussion}

Figure 1 shows examples of changes in the monomer spectrum over time after exposure (20s) with halogen source - EliparTrilight (ESPE, Germany).Polymerization of the tested resins consists of three different stages - very fast changes in time from 10-40s (DC: 25-50\%), medium (5- 25 min, DC: 40-60\%) and very slow (days, weeks from DC: 60 -70). Polymerization studies of the same optical layers of the resin placed between the $\mathrm{KBr}$ crystals allow direct DC determination without the use of an internal standard. The presented method of measurement and analysis allows to accurate compare of polymerization or kinetics or DC and understanding influence of factors such as irradiance, monomer layer thickness, exposure time, type of source, post-polymerization processes in any time and under various conditions.
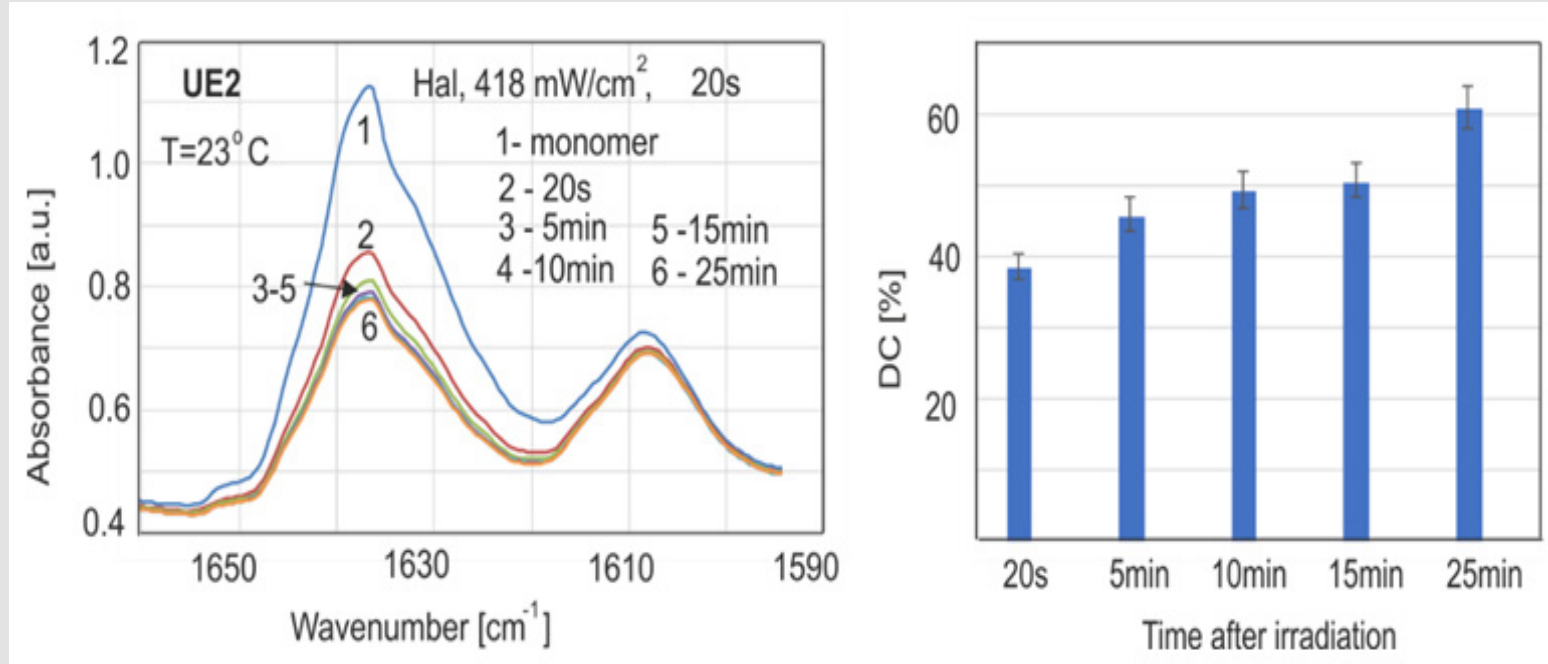

Figure 1: Example of kinetics polymerization.

\section{References}

1. Frauscher K, Ilie N (2013) Degree of conversion of nano-hybrid resinbased composites with novel and conventional matrix formulation. Clin Oral Invest 17(2): 635-642.

2. Ilie N, Obermaier J, Durner J (2014) Effect of modulated irradiation time on the degree of conversion and the amount of elutable substances from nano-hybrid resin-based composites. Clin Oral Invest 18(1): 97-106.
3. Segreto DR, Naufel FS, Brandt WC, Guiraldo RD (2016) Influence of photoinitiator and light-curing source on bond strenth of experimental resin cements to dentin. Braz Dent J 27(1): 83-89.

4. Borges AFS, Chase MA, Guggiar AL, Gonzales MJ (2013) A critical review on the conversion degree of resin monomers by direct analyses. Braz Dent Sci 16(1): 18-26

\section{ISSN: 2574-1241}

DOI: $10.26717 /$ BJSTR.2020.30.004896

Aneta Bombalska. Biomed J Sci \& Tech Res

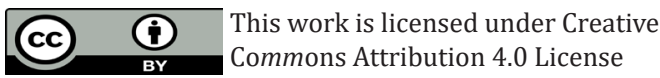

Submission Link: https://biomedres.us/submit-manuscript.php

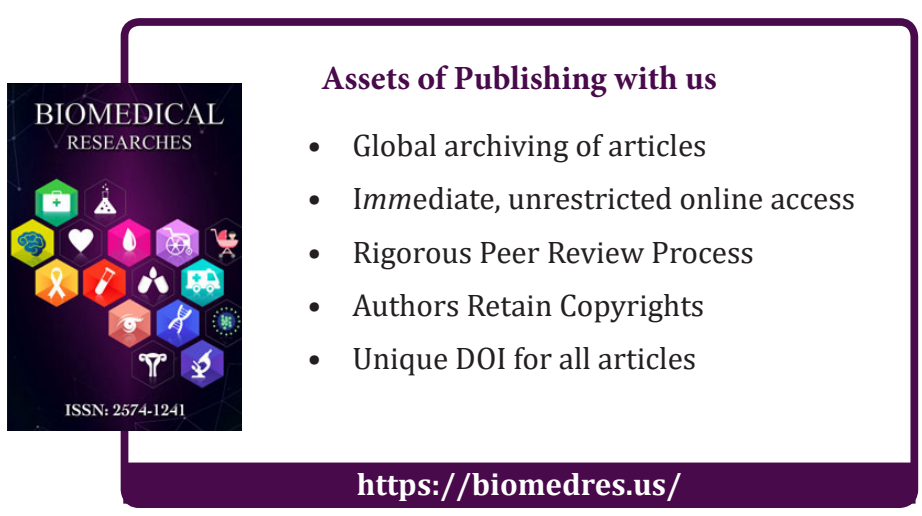

